\title{
Oribátidos, colémbolos y hormigas como indicadores de perturbación del suelo en sistemas de producción agrícola
}

\section{Oribatids, collembola and ants as indicators of soil disturbance in agricultural production systems}

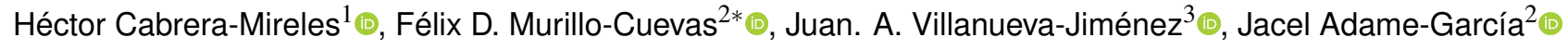 \\ ${ }^{1}$ INIFAP, CIRGOC, Campo Experimental Cotaxtla, km. 34.5 carr. federal Veracruz - Córdoba, municipio de Medellín de Bravo, Veracruz, \\ México. \\ ${ }^{2}$ Tecnológico Nacional de México/l.T. Úrsulo Galván, km 4.5 carr. Cardel-Chachalacas, Úrsulo Galván, Veracruz, México. \\ ${ }^{3}$ Colegio de Postgraduados, Campus Veracruz, km. 88.5 carr. Federal Xalapa-Veracruz, Predio Tepetates, Manlio Fabio Altamirano, \\ Veracruz, México. \\ *Autor de correspondencia: felix.murillo.itug@gmail.com
}

\begin{abstract}
Artículo científico recibido: 11 de octubre de 2018 aceptado: 13 de febrero de 2019
\end{abstract}
RESUMEN. Se comparó la abundancia, riqueza y diversidad de oribátidos, colémbolos y hormigas como indicadores de perturbación del suelo encontrados bajo sistemas de manejo mínimo (tradicional), manejo en transición y manejo tecnificado del cultivo de mango, así como el sistema de manejo tecnificado de la caña de azúcar en la región Centro de Veracruz, México. En cada sistema de manejo se tomaron muestras de suelo y hojarasca, para colectar artrópodos mediante el método de embudos de Berlese-Tullgren. Los muestreos se realizaron en primavera, verano e invierno durante el $2017 \mathrm{y}$ 2018. Las especies con mayor abundancia fueron los ácaros Ceratozetes sp., Archegozetes longisetosus, Scheloribates laticlava, Paropanolophus sp. y Pseudoparasitus sp., los colémbolos Pseudosinella sp. y Seira sp., y las hormigas Solenopsis conjurata, Solenopsis terricola, Pyramica brevicornis y Pachycondyla harpax. Los colémbolos y hormigas fueron más abundantes bajo el sistema de manejo mínimo seguido por manejo en transición, y por último en los sistemas de manejo tecnificado de mango y caña de azúcar. En cambio, los oribátidos fueron más abundantes en el sistema de manejo tecnificado del cultivo de mango. El sistema de manejo tecnificado con caña de azúcar con promedio de 12.3 individuos, 1.3 especies y 0.26 de diversidad fue significativamente inferior a los otros sistemas (abundancia, $F_{3,112}=7.2, p=0.0001$; riqueza, $F_{3,112}$ $=13.3, p=0.0001$; diversidad, $\left.F_{3,112}=13.2, p=0.0001\right)$, los cuales no presentaron diferencias significativas entre sí $(p>$ 0.05).

Palabras clave: Agroecosistemas, fauna edáfica, microartrópodos impacto ambiental, suelo.

ABSTRACT. The abundance, richness and diversity of oribatids, collembola and ants were compared as indicators of soil disturbance found under (traditional) minimal management systems, transition management and technified handling of the mango crop, as well as in the technified management system of sugarcane in the Central Veracruz region in Mexico. In each management system, soil and litter samples were taken, in which arthropods were collected using the Berlese-Tullgren funnels method. The samplings were carried out in three seasons of the spring, summer and winter for 2017 and 2018. The most abundant species were the mites Ceratozetes sp., Archegozetes longisetosus, Scheloribates laticlava, Paropanolophus sp. and Pseudoparasitus sp., Collembola Pseudosinella sp. and Seira sp., and the ants Solenopsis conjurata, Solenopsis terricola, Pyramica brevicornis and Pachycondyla harpax. Colémbolos and ants were more abundant under the minimum management system followed by management in transition, and finally in the systems of technified management of mango and sugarcane. On the other hand, the oribatids were more abundant in the management system of mango cultivation. The management system technified with sugar cane had an average of 12.3 individuals, 1.3 species and amount of 0.26 diversity, which was significantly lower than the other systems (abundance, F3,112 $=7.2, p=0.0001$, richness, F3,112 $=13.3, p=$ 0.0001 , diversity, $F 3,112=13.2, p=0.0001)$, which did not show significant differences among themselves $(p>0.05)$.

Key words: Agroecosystems, edaphic fauna, microarthropods, environmental impact, soil. 


\section{INTRODUCCIÓN}

Los artrópodos que habitan en el suelo desempeñan un papel importante en los servicios ecosistémicos, participan en las interacciones entre los procesos físicos, químicos y biológicos. Las funciones de los artrópodos edáficos muestran que son indicadores de la calidad del suelo y un recurso que debe manejarse de forma adecuada para mejorar los servicios proporcionados a los agroecosistemas (Zagatto et al. 2017). Los ácaros oribátidos e insectos colémbolos y hormigas son los grupos faunísticos más importantes que habitan en el suelo, debido a su abundancia, diversidad y funciones. Estos grupos intervienen en la regulación de poblaciones fúngicas y bacterianas, reciclamiento de minerales, procesos de descomposición, fragmentación de residuos vegetales y creación de bioporos (Norton y BehanPelletier 2009). También promueven la humificación y son componentes de numerosas cadenas tróficas (Giraldo-Mendoza 2014).

Los oribátidos y colémbolos son indicadores biogeográficos y ecológicos por su gran aptitud para la especiación, ciclos de vida cortos y poca dispersión (Palacios-Vargas 2003). También son indicadores de contaminantes ambientales (Gergócs y Hufnagel 2017) y se utilizan como bioindicadores de perturbación en la agricultura y ecosistemas forestales, (Flores-Pardavé et al. 2011). Las hormigas son organismos que tienen gran capacidad de sobrevivir en suelos agrícolas, a pesar de las alteraciones, lo que les permite una alta prevalencia en abundancia y resistencia en sistemas que tienen algún nivel de intervención antrópica (Chanatásig-Vaca et al. 2011). La abundancia y diversidad de artrópodos del suelo se ha utilizado para señalar el impacto de prácticas agrícolas (Cabrera et al. 2011, Lang-Ovalle et al. 2011), por ejemplo en los cultivos agrícolas se sabe que después de una perturbación, puede ocurrir una reducción selectiva de la diversidad de artrópodos (Salas-López et al. 2018).

El uso de suelo en la producción agrícola es uno de los factores claves que afecta la biodiversidad edáfica, con impactos negativos en las propiedades físicas, químicas y biológicas del suelo, provocando su disminución general o pérdida total (Lang-Ovalle et al. 2011, Franco et al. 2016). En la región centro del estado de Veracruz, la agricultura intensiva ha perdido la mayor parte de la cubierta forestal, sustituyendo el cultivo de la caña de azúcar a la vegetación arbórea, lo que afecta de forma negativa la biodiversidad del suelo, entre otros efectos (Gobierno del Estado de Veracruz 2016). El cultivo de mango es uno de los sistemas de producción más importantes, con una superficie cultivada de 17 688 ha (SIAP 2018) bajo diferentes niveles tecnológicos de producción, por lo que es un posible factor de reducción de la biodiversidad de los artrópodos en el suelo (Cabrera et al. 2011, Lang-Ovalle et al. 2011). En los municipios de Actopan y Tierra Blanca es importante la producción de mango (11 064.00 y 2413.00 t, respectivamente) y caña de azúcar (552 200.00 y 1225846.52 t, respectivamente). En Actopan se siembran alrededor de 4037 ha de mango y 6275 ha de caña de azúcar y en Tierra Blanca 1132 ha de mango y 20073 ha de caña de azúcar (SIAP 2018).

Los efectos negativos sobre la biodiversidad de artrópodos del suelo tienen un gradiente de intensificación por las prácticas agrícolas, desde huertos de mango con sistema de manejo mínimo (tradicional), en transición e intensivo (tecnificado), hasta aquellos huertos que han sido sustituidos por caña de azúcar. Con base en lo anterior, el objetivo fue determinar la abundancia, riqueza y diversidad de oribátidos, colémbolos y hormigas como indicadores de perturbación del suelo en diferentes niveles de manejo del cultivo de mango Manila y caña de azúcar en la región centro del estado de Veracruz, México.

\section{MATERIALES Y MÉTODOS}

\section{Área de estudio y sistemas de producción}

El estudio se realizó en dos localidades productoras de mango manila en la región centro del estado de Veracruz, México. Las cuales fueron: 1) Los Ídolos, municipio de Actopan $19^{\circ} 25.705^{\prime} \mathrm{LN}$ y $96^{\circ}$ 31.442' LO con clima $A w_{0}{ }_{0}(w)(i)$ (el menos húmedo de los cálidos subhúmedos con lluvias en verano, con un porcentaje de lluvia invernal menor al $5 \%$ ), os- 
cilación térmica anual menor a $5^{\circ} \mathrm{C}$ (isotermal), precipitación media anual de $860.1 \mathrm{~mm}$ y temperatura media anual de $24.8^{\circ} \mathrm{C}$; y 2) El Pantano, $18^{\circ} 37.983^{\prime}$ LN y $96^{\circ} 16.599^{\prime}$ LO municipio de Tierra Blanca con clima $A w{ }_{2}(w)(e) g$ (el más húmedo de los cálidos subhúmedos con lluvias en verano), oscilación térmica extremosa con temperaturas mayores en el primer semestre del año (García 1987).

En ambas localidades se seleccionaron huertos de mango Manila con los siguientes sistemas de producción agrícola: 1) Manejo tecnificado (S1) en el que se utiliza maquinaria agrícola de forma intensiva, así como aplicación de fertilizantes, insecticidas, fungicidas, herbicidas e inductores de floración, entre otros insumos. 2) Manejo en transición (S2) con uso escaso o nulo de maquinaria e insumos agrícolas. 3) Manejo mínimo tradicional (S3) sin uso de maquinaria e insumos agrícolas, con especies como Rhynchosia minima (L.) DC., Cucurbita argyrosperma K. Kock, Cardiospermum halicacabum Linn, Ipomoea sp., Cardiospermum halicacabum L., Achyranthes indica (L.) Mill, Blechum brownei (Lam.) Urb., Scleria pterota K. Presl ex C.B. Clarke, Cyperus rotundus L., Acalypha alopecuroides Jacq., Acrocomia sp., Vigna lasiocarpa (Mart. ex Benth.) Verdc., Malvaviscus arboreus Cav., Tragia mexicana Müll.Arg., Emilia sonchifolia (L.) DC. ex Wight, Philodendron sp., Acacia cornigera (L.) Willd. y plántulas de Mangifera indica L.; y 4) Sistema de manejo tecnificado de la caña de azúcar (S4), donde por más de 50 años habían sido huertos de mango Manila y su sustitución ocurrió en los últimos cinco años, con poca riqueza de especies vegetales.

\section{Muestreo de artrópodos}

Los muestreos se realizaron en la primavera, verano e invierno, del 2017 y 2018. En cada sistema de producción se seleccionaron dos sitios de colecta, para la obtención de la muestra, se realizó una excavación de $25 \times 25 \times 30 \mathrm{~cm}$ en cada punto de muestreo. Los monolitos de suelo obtenidos, se dividieron en dos estratos sucesivos (hojarasca y 0-30 $\mathrm{cm})$. Las muestras se colocaron en bolsas de plástico, etiquetaron y transportaron al laboratorio para su posterior procesamiento.

\section{Procesamiento de muestras}

Las muestras, se depositaron por separado en embudos de Berlese-Tullgren; se expusieron a una intensidad de calor de $60 \mathrm{~W}$ por $30 \mathrm{~h}$; en la parte inferior de los embudos se colocaron frascos de vidrio con alcohol al $70 \%$ como medio de conservación de los organismos que salían del embudo por efecto del calor. La identificación taxonómica y el conteo de artrópodos extraídos se llevó a cabo con un microscopio estereoscópico. Otros organismos se procesaron en preparaciones permanentes previa identificación a nivel de orden, familia, género y especie. Algunos organismos fueron identificados por especialistas del grupo y con el apoyo de claves taxonómicas de Krantz (1975), Triplehorn y Johnson (2005) y Mackay y Mackay (2014).

\section{Diseño del experimento}

Se utilizó un arreglo factorial en un diseño completamente aleatorio, considerando cinco factores: (A) Año de estudio, con dos niveles: a1) año 2017 y a2) año 2018; (B) Época del año, con tres niveles: b1) primavera, b2) verano e b3) invierno; (C) Localidad, con dos niveles: c1) Los Ídolos y c2) El Pantano; (D) Sustrato, con dos niveles: d1) hojarasca y d2) suelo y (E) El sistema de manejo de mango Manila, con cuatro niveles: e1) tecnificado cultivo de mango, e2) en transición, e3) mínimo tradicional y e4) tecnificado del cultivo caña de azúcar. Las variables de respuesta fueron la abundancia, riqueza y diversidad de especies de artrópodos.

\section{Análisis de datos}

Se calculó la abundancia con el número de organismos de cada especie, la riqueza con el número de especies, la diversidad con el índice de ShannonWiener ( $\left.\mathrm{H}^{\prime}\right)$, utilizando el programa EstimateS versión 8.2.0. Para el análisis estadístico, se realizó un análisis de varianza para las variables de respuesta abundancia, riqueza y diversidad por factor e interacción y se compararon las medias (Tukey, $p \leq 0.05$ ), con el programa SAS v 9.2 . 
Cabrera-Mireles et al.

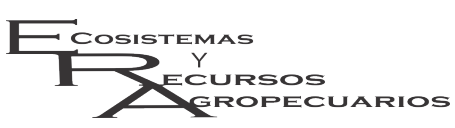

Indicadores biológicos en mango y caña

Ecosist. Recur. Agropec.

6(17):231-241,2019

\section{RESULTADOS}

Al comparar los años de estudio, la abundancia de los artrópodos no registró diferencias significativas ( $p>0.05)$; pero en el primer año registró mayor riqueza (3.8) y diversidad (0.75) de especies, valor que fue diferentes $\left(F_{1,112}=8.3, p=0.0044 ; F_{1,112}\right.$ $=12.7, p=0.0004)$. Las épocas de estudio registraron diferencias en abundancia $(F 2,112=10.0, p=$ $0.0001)$, riqueza $\left(F_{2,112}=3.8, p=0.0241\right)$ y diversidad $\left(F_{2,112}=5.4, p=0.0051\right)$ de especies, siendo verano la época de mayor abundancia de organismos (34.7 individuos) en comparación con la primavera (20.2 individuos) e invierno (12.9 individuos). En primavera se registró en promedio la mayor diversidad (0.79) y riqueza (3.6) de especies, valores que son diferentes a los que se obtuvieron en verano e invierno. La localidad Tierra Blanca con promedio de 28.9 individuos, 3.7 especies y 0.74 de diversidad fue superior a la localidad Los ídolos (18.9 individuos, 2.6 especies y 0.52 diversidad) en todas las variables ecológicas (abundancia, $F_{1,112}=10.7, p=0.0012$; riqueza, $F_{1,112}=9.9, p=0.0018 ;$ diversidad, $F_{1,112}$ $=7.3, p=0.0072$ ). Al comparar los sustratos, se observaron diferencias en la abundancia de organismos $\left(F_{1,112}=20.3, p=0.0001\right)$, siendo la hojarasca el sustrato con mayor abundancia de organismos (33.7 individuos). El sistema de manejo tecnificado con caña de azúcar con 12.3 individuos, 1.3 especies y 0.26 de diversidad fue inferior a los otros sistemas (abundancia, $F_{3,112}=7.2, p=0.0001$; riqueza, $F_{3,112}=13.3, p$ $=0.0001$; diversidad, $\left.F_{3,112}=13.2, p=0.0001\right)$, los cuales no presentaron diferencias entre sí ( $p>0.05)$.

Se colectaron 7701 ejemplares de artrópodos habitantes de la meso y macrofauna del suelo y hojarasca, de los cuales 4367 fueron ácaros, 1 129 colémbolos, 937 hormigas, y 1268 ejemplares de otros artrópodos (Tabla 1). El mayor número de ácaros se registró en el sistema tecnificado del cultivo de mango, en los dos sustratos de estudio (suelo y hojarasca), a diferencia de los colémbolos y hormigas que abundaron más en el sistema de manejo mínimo tradicional. El sistema de manejo tecnificado del cultivo de caña de azúcar, registró la menor abundancia de estos organismos y en general de todos los grupos de artrópodos, destacando thysanopteros, isópteros, pseudoescorpiones, chilopodos, ortópteros y zorápteros.

Del total de ejemplares colectados, el $66 \%$ se obtuvieron de la hojarasca (5 083 individuos) y el 34\% (2 618 individuos) del suelo. En hojarasca, el sistema de manejo mínimo tradicional registró la mayor abundancia de artrópodos (1 942 individuos), seguido del sistema tecnificado del cultivo de mango, en transición y el manejo tecnificado de caña de azúcar. La menor riqueza y diversidad de especies se registró en el sistema de manejo tecnificado de caña de azúcar (16 especies y 0.7 de diversidad). En suelo la mayor abundancia se tuvo en los sistemas mínimo tradicional y en transición (885 individuos); la menor riqueza en el sistema de manejo tecnificado de caña de azúcar (21 especies) y diversidad en los sistemas tecnificados de mango y caña de azúcar (2.4).

El grupo más abundante de ácaros fue el Oribatidae (3 504 individuos), seguido de Gamasidae (434 individuos) y Prostigmata (429 individuos). Se identificaron tres subórdenes, 13 familias, 14 géneros y seis especies de ácaros; el ácaro Ceratozetes sp. (Figura 1a) fue el más abundante, seguida de Archegozetes longisetosus (Figura 1b), los cuales abundaron más en el sistema tecnificado de mango en la hojarasca y suelo. La especie Pseudoparasitus sp. (Figura 1c), fue importante en abundancia, con mayor presencia en el sistema mínimo tradicional; otra especie abundante fue Paropanolophus sp. (Figura 1d) que tuvo su mayor registro en el sistema mínimo tradicional en el sustrato hojarasca y en el sistema en transición en el sustrato suelo. La menor abundancia y riqueza de especies de ácaros se tuvo en el sistema de manejo tecnificado de caña de azúcar, en ambos sustratos (Tabla 2).

En los colémbolos se determinaron 5 familias y 5 géneros. Los colémbolos Pseudosinella sp. y Seria sp. (Figura 2) fueron los de mayor abundancia y presencia en el sistema mínimo tradicional en los dos sustratos; el sistema de manejo tecnificado con caña de azúcar, tuvo la menor abundancia de especies. Mientras que en el sustrato hojarasca se ausentaron las familias Onychiuridae e Isotomidae (Tabla 3).

Para las hormigas se identificaron 7 especies, 
Cabrera-Mireles et al.

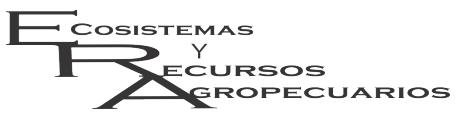

Indicadores biológicos en mango y caña

Ecosist. Recur. Agropec.

6(17):231-241,2019

Tabla 1. Número total de organismos por grupos de meso y macrofauna del suelo y hojarasca en los sistemas de manejo ${ }^{1}$ en la región centro de Veracruz, México.

\begin{tabular}{|c|c|c|c|c|c|c|c|c|}
\hline & \multicolumn{4}{|c|}{ Hojarasca } & \multicolumn{4}{|c|}{ Suelo } \\
\hline & S1 & $\mathrm{S} 2$ & S3 & S4 & $\mathrm{S} 1$ & S2 & S3 & S4 \\
\hline Acarida & 1087 & 617 & 842 & 530 & 467 & 403 & 349 & 72 \\
\hline Collembola & 118 & 45 & 255 & 21 & 135 & 231 & 303 & 21 \\
\hline Hymenoptera (Formicidae) & 66 & 188 & 464 & 0 & 29 & 81 & 107 & 2 \\
\hline Coleoptera & 51 & 46 & 101 & 9 & 36 & 76 & 38 & 4 \\
\hline Psocoptera & 112 & 41 & 152 & 11 & 5 & 5 & 3 & 21 \\
\hline Diplopoda & 30 & 12 & 51 & 1 & 15 & 3 & 20 & 3 \\
\hline Isopoda & 33 & 1 & 25 & 1 & 19 & 15 & 11 & 2 \\
\hline Araneae & 20 & 24 & 30 & 1 & 2 & 9 & 2 & 3 \\
\hline Thysanoptera & 30 & 6 & 20 & 0 & 1 & 3 & 17 & 0 \\
\hline Hemiptera & 6 & 5 & 2 & 0 & 4 & 0 & 18 & 9 \\
\hline Isoptera & 1 & 0 & 0 & 0 & 1 & 32 & 0 & 0 \\
\hline Pseudoscorpionida & 0 & 9 & 0 & 2 & 3 & 14 & 5 & 0 \\
\hline Chilopoda & 6 & 1 & 0 & 0 & 1 & 10 & 9 & 0 \\
\hline Orthoptera & 1 & 0 & 0 & 0 & 1 & 2 & 1 & 0 \\
\hline Zoraptera & 0 & 1 & 0 & 0 & 0 & 1 & 2 & 0 \\
\hline Abundancia & 1561 & 996 & 1942 & 576 & 719 & 885 & 885 & 137 \\
\hline *Riqueza & 53 & 54 & 48 & 16 & 41 & 56 & 45 & 21 \\
\hline **Diversidad & 1.9 & 2.6 & 2.5 & 0.7 & 2.4 & 2.8 & 2.7 & 2.4 \\
\hline
\end{tabular}

${ }^{1}$ Sistemas de manejo: S1-Tecnificado con mango; S2-Transición; S3-Mínimo; S4-Tecnificado con caña de azúcar. ${ }^{*}$ Número de individuos, ${ }^{* *}$ Número de morfoespecies, ${ }^{* \star *}$ Índice de Shannon-Weaver.
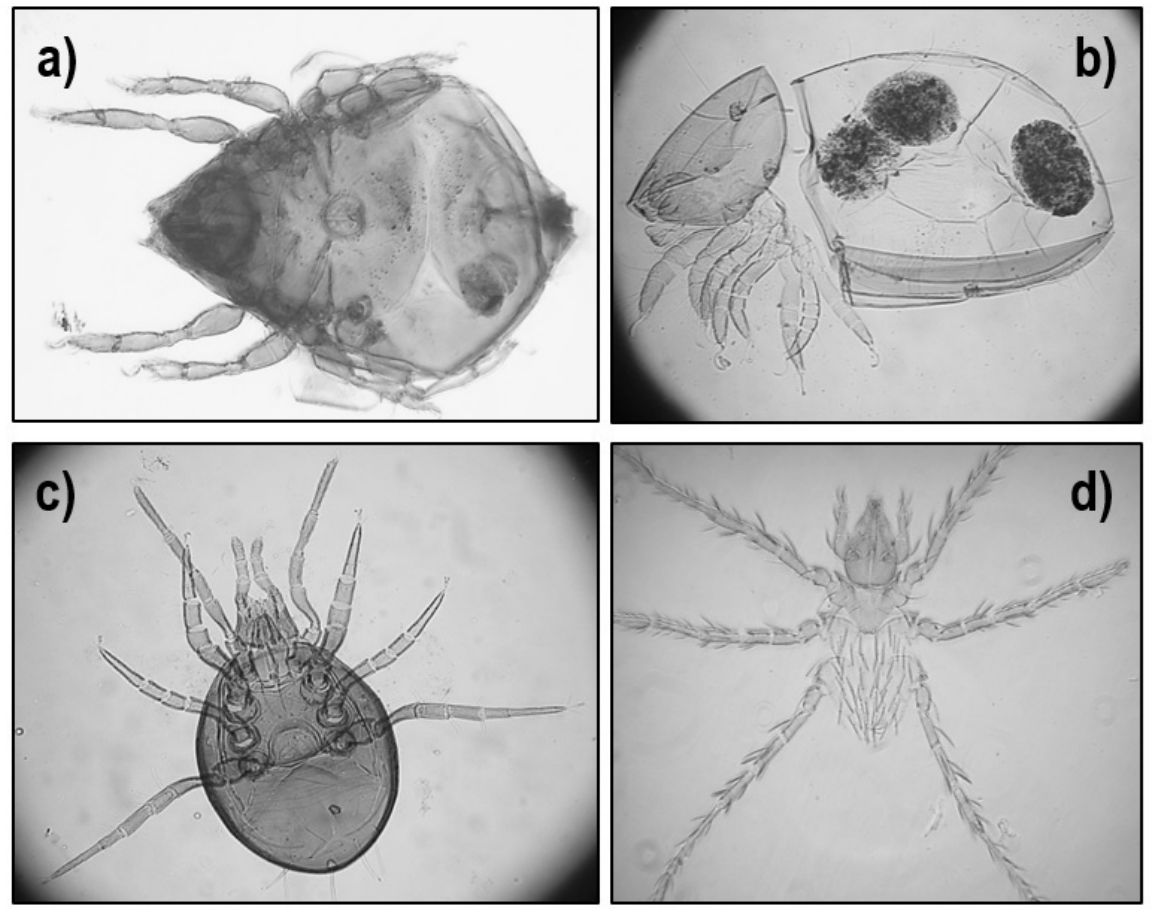

Figura 1. Especies de ácaros más importantes colectados en hojarasca y suelo en sistemas de manejo de mango Manila en la región centro de Veracruz, México. a)Ceratozetes sp., b)Archegozetes longisetosus, c) Pseudoparasitus sp., d) Paropanolophus sp.

Solenopsis conjurata (Figura 3a) y S. terricola (Figura $3 b)$ fueron las de mayor abundancia; los sistemas de manejo mínimo tradicional y en transición fueron los que tuvieron mayor presencia de estas especies en 
Cabrera-Mireles et al.

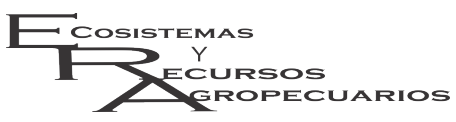

Indicadores biológicos en mango y caña

Ecosist. Recur. Agropec.

6(17):231-241,2019

Tabla 2. Lista faunística de ácaros colectados en hojarasca y suelo en sistemas de manejo $^{1}$ en la región centro de Veracruz, México.

\begin{tabular}{|c|c|c|c|c|c|c|c|c|}
\hline & \multicolumn{4}{|c|}{ Hojarasca } & \multicolumn{4}{|c|}{ Suelo } \\
\hline & S1 & S2 & S3 & S4 & S1 & S2 & S3 & S4 \\
\hline $\begin{array}{l}\text { ORIBATIDAE } \\
\text { Ceratozetidae }\end{array}$ & & & & & & & & \\
\hline $\begin{array}{l}\text { Ceratozetes sp. } \\
\text { Tryphochthoniidae }\end{array}$ & 606 & 211 & 271 & 478 & 255 & 149 & 113 & 17 \\
\hline $\begin{array}{l}\text { Archegozetes longisetosus } \\
\text { Eupthyracaridae }\end{array}$ & 410 & 230 & 296 & 3 & 86 & 82 & 48 & 18 \\
\hline Scheloribates laticlava & 22 & 8 & 18 & 13 & 10 & 18 & 4 & 8 \\
\hline $\begin{array}{l}\text { Rhysotritia clavata } \\
\text { Eremulidae }\end{array}$ & 2 & 4 & 7 & 0 & 0 & 0 & 3 & 0 \\
\hline Eremulus rigidisetosus & 1 & 0 & 2 & 29 & 0 & 10 & 6 & 0 \\
\hline $\begin{array}{l}\text { Oppiidae } \\
\text { Galumnidae }\end{array}$ & 0 & 2 & 1 & 0 & 2 & 19 & 14 & 1 \\
\hline $\begin{array}{l}\text { Galumna sp. } \\
\text { Malaconothridae }\end{array}$ & 1 & 2 & 1 & 0 & 12 & 0 & 10 & 0 \\
\hline $\begin{array}{l}\text { Malaconothrus sp. } \\
\text { PROSTIGMATA } \\
\text { Erythraeidae }\end{array}$ & 0 & 0 & 0 & 0 & 1 & 0 & 0 & 0 \\
\hline Paropanolophus sp. & 8 & 54 & 124 & 0 & 1 & 38 & 0 & 0 \\
\hline Leptus sp. & 0 & 19 & 1 & 0 & 0 & 0 & 0 & 0 \\
\hline $\begin{array}{l}\text { Balaustium sp. } \\
\text { Cheyletidae }\end{array}$ & 0 & 0 & 0 & 0 & 0 & 1 & 0 & 0 \\
\hline $\begin{array}{l}\text { Cheletogenes sacalow } \\
\text { Smarididae }\end{array}$ & 6 & 7 & 15 & 0 & 64 & 16 & 45 & 24 \\
\hline $\begin{array}{l}\text { Smaris sp. } \\
\text { GAMASIDAE } \\
\text { Laelapidae }\end{array}$ & 0 & 0 & 0 & 0 & 6 & 0 & 0 & 0 \\
\hline Pseudoparasitus sp. & 27 & 75 & 106 & 7 & 29 & 56 & 96 & 4 \\
\hline $\begin{array}{l}\text { Phytoseiidae } \\
\text { Macrochelidae }\end{array}$ & 4 & 0 & 0 & 0 & 1 & 14 & 10 & 0 \\
\hline Macrocheles insignitus & 0 & 5 & 0 & 0 & 0 & 0 & 0 & 0 \\
\hline
\end{tabular}

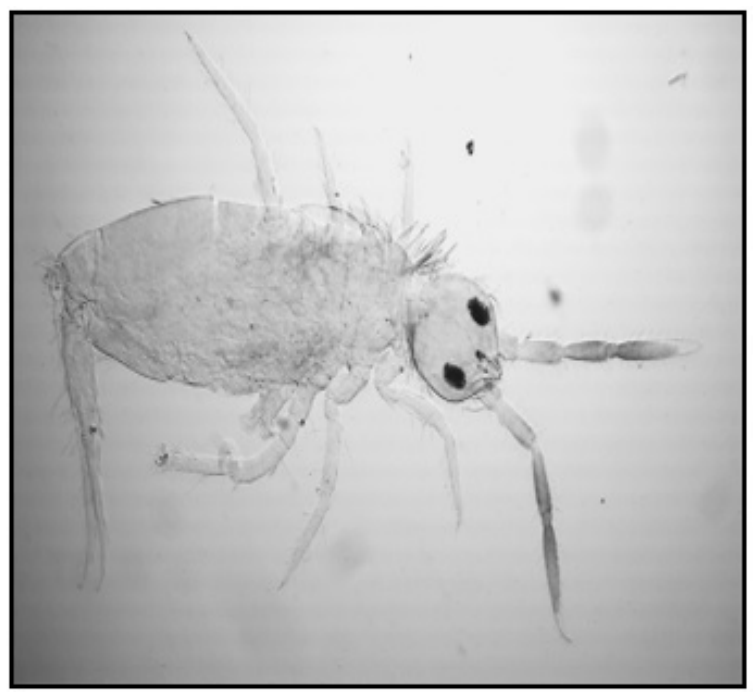

Figura 2. Colémbolo Seria sp., colectado en hojarasca y suelo en sistemas de manejo de mango Manila en la región centro de Veracruz, México. 
Tabla 3. Lista faunística de colémbolos colectados en hojarasca y suelo en sistemas de manejo ${ }^{1}$ en la región centro de Veracruz, México.

\begin{tabular}{|c|c|c|c|c|c|c|c|c|}
\hline & \multicolumn{4}{|c|}{ Hojarasca } & \multicolumn{4}{|c|}{ Suelo } \\
\hline & $\mathrm{S} 1$ & S2 & S3 & S4 & S1 & S2 & S3 & S4 \\
\hline Entomobrydae & & & & & & & & \\
\hline Pseudosinella sp. & 102 & 31 & 130 & 19 & 59 & 188 & 247 & 18 \\
\hline $\begin{array}{l}\text { Seira sp. } \\
\text { Sminthuridae }\end{array}$ & 11 & 5 & 120 & 1 & 43 & 12 & 33 & 3 \\
\hline $\begin{array}{l}\text { Jannenotia sp. } \\
\text { Tomoceridae }\end{array}$ & 5 & 7 & 5 & 1 & 30 & 9 & 9 & 0 \\
\hline Pongonognathelus sp. & 0 & 2 & 0 & 0 & 0 & 9 & 0 & 0 \\
\hline $\begin{array}{l}\text { Onychiuridae } \\
\text { Isotomidae }\end{array}$ & & & & & 3 & 12 & 14 & 0 \\
\hline Folsomia sp. & & & & & 0 & 1 & 0 & 0 \\
\hline
\end{tabular}


Figura 3. Especies de hormigas más importates colectadas en hojarasca y suelo en sistemas de manejo de mango Manila en la región centro de Veracruz, México. a)Solenopsis conjurata, b) Solenopsis terricola.

los dos sustratos; el sistema de manejo tecnificado con caña de azúcar y tecnificado con mango registraron la menor abundancia y mayor ausencia de especies. En el sustrato suelo se ausento la especie Odontomachus laticeps y en hojarasca fue mínima su presencia (Tabla 4).

En el sustrato hojarasca, el sistema de manejo mínimo tradicional tuvo la mayor abundancia de organismos compuestos por ácaros, colémbolos y hormigas (1 561 individuos), y sólo el sistema en transición fue similar a éste en riqueza (19 especies), diversidad (2.08) y equidad (0.62) de especies. Las equidades de estos dos sistemas de manejo muestran que las especies se encuentran bien distribuidas en relación a sus abundancias. Estos dos sistemas de manejo presentaron mayor similitud $(0.73 \%)$ con respecto a los sistemas de manejo restantes.

En el sustrato suelo, los valores ecológicos fueron muy similares al sustrato hojarasca, siendo el sistema mínimo tradicional el de mayor abundan- cia (759 individuos), y el sistema en transición el de mayor riqueza (19 especies), diversidad (2.31) y equidad (0.69). En este sustrato, al igual que en suelo, las equidades registradas en estos dos sistemas de manejo demuestran una buena distribución de las especies. En relación a la similitud estos dos sistemas tuvieron uno de los valores más altos $(0.61 \%)$, solo menor por la similitud del sistema mínimo tradicional y tecnificado con mango $(0.68 \%)$. El sistema de manejo tecnificado con caña de azúcar registro los valores ecológicos más bajos en los dos sustratos y la similitud más baja $(0.35-0.50 \%)$ al compararlo con los restantes sistemas de manejo de mango Manila.

\section{DISCUSIÓN}

En los muestreos de suelo y hojarasca de los diferentes sistemas de manejo de mango Manila se observó la presencia de ácaros, colémbolos y hormi- 
Cabrera-Mireles et al.

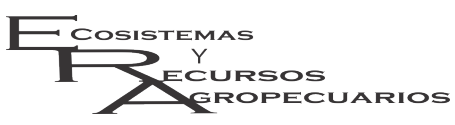

Indicadores biológicos en mango y caña

Ecosist. Recur. Agropec.

6(17):231-241,2019

Tabla 4. Lista faunística de hormigas colectadas en hojarasca y suelo en sistemas de manejo ${ }^{1}$ en la región centro de Veracruz, México.

\begin{tabular}{lcccccccc}
\hline & \multicolumn{4}{c}{ Hojarasca } & \multicolumn{4}{c}{ Suelo } \\
\cline { 2 - 10 } & S1 & S2 & S3 & S4 & S1 & S2 & S3 & S4 \\
\hline Solenopsis conjurata & 49 & 106 & 387 & 0 & 14 & 44 & 81 & 2 \\
Solenopsis terricola & 0 & 54 & 70 & 0 & 7 & 28 & 7 & 0 \\
Pyramica brevicornis & 0 & 27 & 0 & 0 & 0 & 9 & 0 & 0 \\
Pachycondyla harpax & 5 & 1 & 5 & 0 & 4 & 0 & 18 & 0 \\
Eurhopalothrix gravis & 11 & 0 & 0 & 0 & 0 & 0 & 1 & 0 \\
Solenopsis geminata & 0 & 0 & 1 & 0 & 4 & 0 & 0 & 0 \\
Odontomachus laticeps & 1 & 0 & 1 & 0 & 0 & 0 & 0 & 0 \\
\hline 'Sistemas de manejo: S1-Tecnificado; S2-Transición; S3-Mínimo; S4-Sustituido \\
por caña de azúcar.
\end{tabular}

gas; por lo que la abundancia, riqueza y diversidad de estos organismos pueden utilizarse como indicador biológico de la calidad del suelo (MamaniMamani et al. 2012, Genoy et al. 2013, Socarrás e Izquierdo 2014). La abundancia, riqueza y diversidad de ácaros, colémbolos y hormigas registrados en cada uno de los sistemas de manejo de mango Manila, permite inferir que el sistema de manejo agrícola afecta la micro y mesofauna edáfica. También permite conocer que el sistema de manejo tecnificado con caña de azúcar impacta de forma significativa a estos organismos, el cual es uno de los sistemas altamente tecnificado y con mayor perturbación ecológica en relación al cultivo de mango (Lang-Ovalle et al. 2011). El cultivo de caña de azúcar se caracteriza por elevado consumo de fertilizantes y plaguicidas, lo que puede afectar las poblaciones de ácaros, colémbolos y hormigas, como lo menciona Socarrás y Robaina (2011) y de Groot et al. (2016) quienes mencionan que la diversidad y abundancia de estos organismos se reduce a medida que se incrementa la intensidad del manejo agrícola. Los resultados indican que la introducción de cultivos y/o técnicas agrícolas como las que se usan en el cultivo de caña de azúcar o sistema de manejo tecnificado de mango, perturban las comunidades de ácaros, colémbolos y hormigas como lo indican Mahdi et al. (2017) y Socarrás y Robaina (2011). Estos resultados coinciden con investigaciones que reportan mayor abundancia de los componentes de la mesofauna del suelo en los ecosistemas boscosos y agroforestales que en los de uso agrícola (Hernández-Vigoa et al. 2018), así como los que comparan los sistemas de producción de mango y caña de azúcar (Lang-Ovalle et al. 2011).

En los sistemas de manejo que incluyen sólo al cultivo de mango Manila, los ácaros fueron más abundantes en el sistema tecnificado, a diferencia de los colémbolos y hormigas, que fueron más abundantes en el sistema mínimo tradicional, lo que indica que es un sistema estable. Estos organismos pueden ser utilizados como bioindicadores de conservación/perturbación de los suelos en los sistemas de manejo de mango Manila, ya que los ácaros como los oribátidos son más abundantes en sistemas perturbados (Socarrás y Robaina 2011, Socarrás e Izquierdo 2014), debido a su capacidad de adaptación a los ambientes alterados (Morais et al. 2010). Por otro lado, los colémbolos son más sensibles que los ácaros, con capacidad de adaptación a distintos ambientes, lo que los hace buenos indicadores edáficos (UribeHernández et al. 2010).

En el sistema de manejo mínimo tradicional, las comunidades de ácaros, colémbolos y hormigas edáficas fueron aproximadamente 3.6 veces superiores en abundancia con relación al sistema de manejo tecnificado por caña de azúcar y de aproximadamente de 1.2 y 1.5 veces más abundantes que el sistema tecnificado y en transición, respectivamente. Lo que indica, predominio de especies de arvenses y características de los árboles en el sistema mínimo tradicional, los cuales son frondosos y de gran porte, por lo que generan abundante materia orgánica por la caída de hojas, con relación $\mathrm{C} / \mathrm{N}$ menor que otras plantas como las gramíneas (Hernández-Vigoa et al. 2018). Por otro lado, la compleja composición florística y mantillo heterogéneo 
típico de los huertos de mango Manila con sistemas de manejo mínimo tradicional, promueve el proceso de descomposición, lo que favorece la diversidad de hongos y bacterias, que son fuentes de alimento de los colémbolos y hormigas. Las familias: oribátidos Ceratozetidae, Eupthyracaridae, Oppiidae y Galumnidae, se reportan en otros cultivos agrícolas como en el ajo y papa, donde se observa efecto negativo sobre la acarofauna del suelo debido a posibles usos indiscriminados de agroquímicos (Vázquez-Rojas y Estrada-Venegas 2010, Genoy et al. 2013). En los colémbolos, la familia Entomobryidae, junto con las especies Pseudosinella sp. y Seria sp., se reportan en cultivos como nopal, maíz, alfalfa y café (FloresPardavé et al. 2011) con mayor riqueza en sistemas menos tecnificados. Las hormigas Solenopsis conjurata, S. terricola y $S$. geminata son especies comunes en suelo de plantaciones cafetales, forestales monoespecíficas de cedro y huertos caseros con cedro y especies frutales (Chanatásig-Vaca et al. 2011, Adolfo et al. 2013). En relación a plantaciones de mango o caña de azúcar es poco lo que se ha documentado con respecto a las especies de ácaros, colémbolos y hormigas, los trabajos realizados se enfocan a grupos taxonómicos superiores de macrofauna edáfica (Lang-Ovalle et al. 2011) y artrópodos asociados al follaje y arvenses (Cabrera et al. 2011). Lo que hace que la información generada sea uno de los primeros reportes de estas especies de ácaros, colémbolos y hormigas en mango y caña de azúcar.

Los efectos del sustrato y época del año sobre la fauna edáfica indican la importancia de estos factores en la abundancia de los organismos, ya que la mayor abundancia de ácaros, colémbolos y hormigas se tuvo en la hojarasca y en verano, lo que indica que el contenido de materia orgánica y la precipitación o humedad son factores que influyen en la densidad poblacional de los ácaros, colémbolos y hormigas (Holmstrup et al. 2017). La importancia de las lluvias y el sustrato hojarasca en los sistemas de manejo de mango Manila puede deberse a que son factores generadores de abundancia biológica, ya que la hojarasca brinda cobertura al suelo, disminuye el efecto negativo de las labores agrícolas, mejora la humedad y temperatura en el medio edáfico, además garantiza una fuente adicional de alimento a los microartrópodos por los exudados radicales de las plantas.

\section{CONCLUSIONES}

Las condiciones de espacio y tiempo en los sistemas de manejo de mango Manila modifican la abundancia, diversidad y riqueza de artrópodos y por consecuencia la función de estos en los sistemas agrícolas. El sistema de manejo tecnificado con caña de azúcar tuvo impacto negativo sobre los artrópodos asociados a suelo y hojarasca. La abundancia de colémbolos y hormigas, grupos indicadores de fertilidad y estabilidad del suelo, fue mayor en el sistema de manejo mínimo tradicional, seguido del sistema en transición, tecnificado de mango y tecnificado con caña de azúcar. Para los oribátidos, grupo indicador de perturbación del medio edáfico, se tuvo la mayor presencia en el sistema de manejo tecnificado con mango. Las lluvias y el sustrato hojarasca fueron las variables ambientales mas relacionadas con la abundancia, riqueza y diversidad.

\section{AGRADECIMIENTOS}

Al Tecnológico Nacional de México por el financiamiento de los proyectos institucionales 6218.17$P$ y 6609.18-P. Al INIFAP CE Cotaxtla por su contribución. A la Dra. Edith G. Estrada Venegas de Colegio de Postgraduados Campus Montecillo por la identificación de las especies Acari y Collembola, Al Dr. Luís N. Quiroz Robledo del Instituto de Ecología, Xalapa, Veracruz (Formicidae), Dr. Juan Francisco Pérez Domínguez, Campo Experimental Centro Altos de Jalisco, Región Pacifico Centro, INIFAP (Formicidae) y Dr. Valentín A. Esqueda Esquivel, Campo Experimental Cotaxtla, INIFAP (Arvenses).

\section{LITERATURA CITADA}

Adolfo ZG, Marcela AL, Chacón De UP (2013) Diversidad de hormigas (Hymenoptera: Formicidae) en un paisaje cafetero de Risaralda, Colombia. Revista Colombiana de Entomología 39: 141-149. 
Cabrera MH, Murillo CFD, Ortega ZDA, Villanueva JJA, Escobar DAA (2011) Impact of mango Manila management systems on arthropods in foliage and weeds. Tropical and Subtropical Agroecosystems 13: 317-326.

Chanatásig-Vaca CI, Huerta LE, Rojas FP, Ponce-Mendoza A, Mendoza VJ, Morón RA, et al. (2011) Efecto del uso de suelo en las hormigas (Formicidae: Hymenoptera) de Tikinmul, Campeche, México. Acta Zoológica Mexicana 27: 441-461.

de Groot GA, Jagers op Akkerhuis GAJM, Dimmers WJ, Charrier X, Faber JH (2016) Biomass and diversity of soil mite functional groups respond to extensification of land management, potentially affecting soil ecosystem services. Frontiers in Environmental Science 4: 1-12.

Flores-Pardavé L, Palacios-Vargas JG, Castaño-Meneses G, Cutz-Pool LGQ (2011) Colémbolos de suelos agrícolas en cultivos de alfalfa y de maíz adicionados con biosólidos en Aguascalientes, México. Agrociencia 45: 353-362.

Franco ALC, Bartz MLC, Cherubin MR, Baretta D, Cerri CEP, Feigl BJ, et al. (2016) Loss of soil (macro) fauna due to the expansion of Brazilian sugarcane acreage. Science of the Total Environment 563-564: 160-168.

García E (1987) Modificaciones al Sistema de Clasificación Climática de Köppen. $4^{\mathrm{a}}$ Ed. Universidad Nacional Autónoma de México. México. 130p.

Genoy JYM, Castillo FJA, Bacca T (2013) Ácaros oribátidos presentes en seis sistemas de uso del suelo en Obonuco, Pasto (Nariño). Boletín Científico Museo de Historia Natural 17: 60-68.

Gergócs V, Hufnagel L (2017) Comparing the natural variation of oribatid mite communities with their changes associated with anthropogenic disturbance. Environmental Monitoring and Assessment 189: 203. Doi: 10.1007/s10661-017-5897-3.

Giraldo-Mendoza AE (2014) Nuevos registros de Blennidus Motschulsky, 1865 y Calleida Dejean, 1825 (Coleoptera: Carabidae) para Perú. Revista Peruana de Entomología 49: 143-148.

Gobierno del Estado de Veracruz (2016) Plan Veracruzano de Desarrollo (2011-2016) Gobierno del Estado de Veracruz. Veracruz. 307p.

Hernández-Vigoa G, Cabrera-Dávila GC, Izquierdo-Brito I, Socarrás-Rivero AA, Hernández-Martínez L, SánchezRendón JA (2018) Indicadores edáficos después de la conversión de un pastizal a sistemas agroecológicos. Pastos y Forrajes 41: 3-12.

Holmstrup M, Damgaard C, Schmidt IK, Arndal MF, Beier C, Mikkelsen TN, Ambus P, et al. (2017) Long-term and realistic global change manipulations had low impact on diversity of soil biota in temperate heathland. Scientific Reports 7. 41388.

Krantz GW (1975) A Manual of Acarology. Oregon State University. Corvallis, Oregon. 335p.

Lang-Ovalle PF, Pérez-Vázquez A, Martínez-Dávila JP, Platas-Rosado DE, Ojeda-Enciso LA, González-Acuña IJ (2011) Macrofauna edáfica asociada a plantaciones de mango y caña de azúcar. Terra Latinoamerica 29: 169-177.

Mahdi JE, Abbott LK, Pauli N, Solaiman ZM (2017) Biological indicators for soil health: potential for development and use of on-farm tests. In: Varma A, Sharma A (eds.) Modern tools and techniques to understand microbes. Switzerland: Springer. Pp: 123-134.

Mamani-Mamani G, Mamani-Pati F, Sainz-Mendoza H, Villca-Huanaco R (2012) Comportamiento de la lombriz roja (Eisenia spp.) en sistemas de vermicompostaje de residuos orgánicos. Journal of the Selva Andina Research Society 3: 44-54. 
Mackay WP, Mackay EE (2014) Clave de los géneros de hormigas en México y América Central (Hymenoptera: Formicidae). Department of Biological Sciences. Laboratory for Environmental Biology. University of Texas, El Paso, TX 79968, E.U. 36p.

Morais JW, Oliveira VS, Dambros CS, Tapia-Coral SC, Acioli ANS (2010) Mesofauna do solo em diferentes sistemas de uso da terra no Alto Rio Solimões, AM Neotropical Entomology 39: 145-152.

Norton RA, Behan-Pelletier VM (2009) Oribatida. En: Krantz GW, Walter DE (Eds.) A manual of acarology. 3rd ed, Texas Tech University Press. Lubbock, Texas. pp: 430-564.

Palacios-Vargas, JG (2003) Los microartrópodos (Collembola) de la selva tropical húmeda. En: Álvarez-Sánchez J, Naranjo-García E (eds.). Ecología del suelo en la selva tropical húmeda de México. Instituto de Ecología, Instituto de Biología. Veracruz, México. 298p.

Salas-López A, Violle C, Mallia L, Orivel J, Didham R (2018) Land-use change effects on the taxonomic and morphological trait composition of ant communities in French Guiana. Insect Conservation and Diversity 11: 162-173.

SIAP (2018) Servicio de Información Agroalimentaria y Pesquera. Anuario Estadístico de la Producción Agrícola, año 2017. SAGARPA. http://infosiap.siap.gob.mx/aagricola_siap_gb/icultivo/index.jsp. Fecha de consulta: 26 de julio 2018.

Socarrás A, Izquierdo I (2014) Evaluación de sistemas agroecológicos mediante indicadores biológicos de la calidad del suelo: mesofauna edáfica. Pastos y Forrajes 37: 47-54.

Socarrás A, Robaina N (2011) Caracterización de la mesofauna edáfica bajo diferentes usos de la tierra en suelo Ferralítico Rojo de Mayabeque y Artemisa. Pastos y Forrajes 34: 185-197.

Triplehorn CA, Johnson NF (2005) Borror and deLong's introduction to the study of insects. 7th ed. Thomson Brooks/Cole. Belmont, California. 864p.

Uribe-Hernández R, Juárez-Méndez C A, Montes de Oca MA, Palacios-Vargas JG, Cutz-Pool L, Mejía-Recarmier BE (2010) Colémbolos (Hexapoda) como bioindicadores de la calidad de suelos contaminados con hidrocarburos en el sureste de México. Revista Mexicana de Biodiversidad 81: 153-16

Vázquez-Rojas IM, Estrada-Venegas EG (2010) Ereynetid mites (Tydeoidea: Ereynetidae) associated with garlic crops in Guanajuato, Mexico. In: Sabelis M, Bruin J (eds) Trends in Acarology. Springer. Dordrecht. pp: 207-210

Zagatto MRG, Niva CC, Thomazini MJ, Baretta D, Santos A, Nadolny H, et al. (2017) Soil invertebrates in different land use systems: How integrated production systems and seasonality affect soil mesofauna communities. Journal of Agricultural Science and Technology 7: 150-161. 
